\title{
Quasideterministic realization of a universal quantum gate in a single scattering process
}

\author{
F. Ciccarello, ${ }^{1,3}$ D. E. Browne, ${ }^{2}$ L. C. Kwek, ${ }^{3}$ H. Schomerus, ${ }^{4}$ M. Zarcone,${ }^{5}$ and S. Bose ${ }^{2}$ \\ ${ }^{1}$ Scuola Normale Superiore, Piazza dei Cavalieri, 7, I-56126 Pisa, Italy \\ ${ }^{2}$ Department of Physics and Astronomy, University College London, Gower Street, London WC1E 6BT, United Kingdom \\ ${ }^{3}$ Centre for Quantum Technologies, National University of Singapore, 3 Science Drive 2, Singapore 117543 \\ ${ }^{4}$ Department of Physics, Lancaster University, Lancaster, LAl 4YB, United Kingdom \\ ${ }^{5}$ CNISM and Dipartimento di Fisica, Università degli Studi di Palermo, Viale delle Scienze, Edificio 18, I-90128 Palermo, Italy
}

(Received 28 July 2011; revised manuscript received 11 November 2011; published 17 May 2012)

\begin{abstract}
We show that a flying particle, such as an electron or a photon, scattering along a one-dimensional waveguide from a pair of static spin- $\frac{1}{2}$ centers, such as quantum dots, can implement a CONTROLLED-Z gate (universal for quantum computation) between them. This occurs quasideterministically in a single scattering event, with no need for any postselection or iteration and without demanding the flying particle to bear any internal spin. We show that an easily matched hard-wall boundary condition along with the elastic nature of the process are key to such performances.
\end{abstract}

DOI: 10.1103/PhysRevA.85.050305

PACS number(s): 03.67.Lx, 03.67.Hk, 42.50.-p

Interfacing static qubits [1] mediated by flying particles is a prominent paradigm in the quest for efficient ways to implement quantum information processing (QIP) $[2,3]$. As a major motivation, this is the only way to jointly address quantum registers located far from each other, thus featuring no direct mutual interaction (this is usually sought to favor local addressing). Within this general framework, over the past few years a research line has thrived around the idea that the crosstalk between the static qubits can be mediated by particles scattering from them [4-15].

Yet, all of such strategies unavoidably face an inherent major drawback. For a given quantum task to be efficiently accomplished, the link between the static objects should occur by means of the local interaction of each static object with a quantum flying bus. Namely, this should feature inherently quantum motional and/or internal (pseudo) spin degrees of freedom (DsOF). To do so, however, the coupling between the flying and static particles will in general entangle them so as to bring about decoherence affecting the DsOF of the static objects. Owing to such effect, the attainment of satisfactory figures of merit thus demands further actions to complement the above interaction. These typically comprise iterated injections of the flying particles and postmeasurements over their DsOF [2-15]. While such bus-dynamics conditioning generally enhances the performances, it usually comes at the cost of making the process probabilistic and may be demanding in practice (e.g., spin postselection of mobile electrons in semiconducting media [16]).

Moreover, as far as scattering scenarios are concerned, there appear to be intrinsic hindrances to accomplishing certain tasks such as the implementation of a two-qubit quantum gate (TQG), typically the most challenging and essential process in most QIP architectures especially when allowing for universal quantum computation (QC). To see this, assume that we need to realize a TQG between a flying qubit and a scattering center (SC) endowed with spin in a one-dimensional (1D) waveguide [17]. Even if the dynamics is conditioned to either the reflection or transmission channel, the resulting process lacks unitarity (a paramount prerequisite for a TQG), unless quite specific regimes of parameters and interaction models are addressed [17]. Analogous considerations a fortiori hold when many scattering centers are present and a gate involving their DsOF is sought, which is our focus in this work. Scattering-based scenarios thus appear as adverse arenas to perform quantum algorithms, which arguably explains why mere entanglement generation has been almost exclusively investigated to date [4-15]. Nevertheless, scattering-based implementations are attractive because of the low demand for control. One normally just needs to set the itinerant-bus wave vector and wait for the collision to occur, thus bypassing any interaction-time tuning (usually a significant noise source). Further benefits such as the resilience to relevant detrimental factors including static disorder [13], phase noise [14], and imperfect particle-wavevector setting [15] have been shown. Except for the attempt in Ref. [17], such advantages have so far been harnessed solely for mere entanglement generation [4-15].

Here, we discover a simple strategy for the realization of quantum gates between static qubits through a particle scattering from them. The injection of the latter, which is not demanded to bear any internal DOF, followed by its multiple scattering from the SCs suffice to quasideterministically achieve the gate in one shot. Also, neither postselection of any kind nor repeated sending of the flying mediators is required. For two static qubits a universal CONTROLLED-Z (CZ) gate naturally arises, showing the effectiveness of our scheme.

Central idea. Consider a monochromatic spinless particle $f$ of wave vector $k$ propagating along a 1D wire that impinges on an array of SCs [see Fig. 1(a)]. Once multiple scattering has occurred, and assuming in particular that the process is elastic, $f$ can only be found either reflected or transmitted with wave vectors $-k$ and $k$, respectively [see Fig. 2(b)]. Let $\{|v\rangle\}$ be a basis of the SCs' Hilbert space and $|\mu\rangle$ one of its elements, whereas $| \pm k\rangle$ are momentum eigenstates of $f$. Let $\left|\Psi_{i n}\right\rangle=|k\rangle|\mu\rangle$ be the overall system's initial state. As $f$ is scattered off, the final state reads

$$
\left|\Psi_{f}\right\rangle=|-k\rangle \sum_{\nu} r_{\nu \mu}|v\rangle+|k\rangle \sum_{\nu} t_{\nu \mu}|v\rangle,
$$

where $r_{v \mu}\left(t_{\nu \mu}\right)$ is a reflection (transmission) probability amplitude corresponding to the initial and final centers' states $|\mu\rangle$ and $|v\rangle$, respectively. Defining a reflection (transmission) operator $\hat{R}(\hat{T})$ in the Hilbert space of the SCs such 

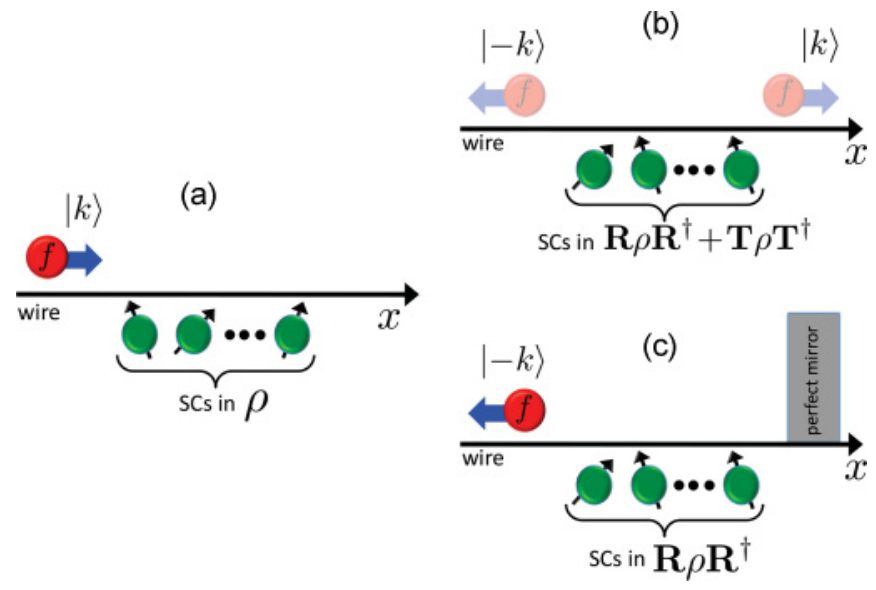

FIG. 1. (Color online) Scheme working principle. (a) $f$ impinges on a set of SCs in $\rho$. (b) After the interaction, $f$ is either reflected or transmitted while the SCs undergo a nonunitary quantum map. (c) With a perfect mirror beyond the centers, $f$ can be only back reflected and the unitary $\hat{R}$ is applied to the SCs.

that $\langle v|\hat{R}| \mu\rangle=\mathbf{R}_{v \mu}=r_{v \mu}\left(\langle\mu|\hat{T}| v\rangle=\mathbf{T}_{v \mu}=t_{v \mu}\right)$, Eq. (1) can be arranged as $\left|\Psi_{f}\right\rangle=|-k\rangle \hat{R}|\mu\rangle+|k\rangle \hat{T}|\mu\rangle$. Tracing over $f$, the final SC's density operator reads $\hat{R}|\mu\rangle\langle\mu| \hat{R}^{\dagger}+$ $\hat{T}|\mu\rangle\langle\mu| \hat{T}^{\dagger}$. Thus when the centers are initially in an arbitrary (in general mixed) state $\rho$ their final state is given by

$$
\rho^{\prime}=\mathbf{R} \rho \mathbf{R}^{\dagger}+\mathbf{T} \rho \mathbf{T}^{\dagger} .
$$

The normalization condition $\sum_{v}\left(\left|r_{v \mu}\right|^{2}+\left|t_{v \mu}\right|^{2}\right)=1 \forall \mu$ entails $\hat{R}^{\dagger} \hat{R}+\hat{T}^{\dagger} \hat{T}=\mathbb{1}$, where $\mathbb{1}$ is the identity operator of the SCs' Hilbert space. We would like the scattering process to implement a multiqubit gate, which is unitary, between the SCs. In general, this is not the case, as is evident from Eq. (2), showing that the SCs undergo instead a quantum map [1] comprising reflection and transmission channels.

Such hindrance can be overcome in a very natural fashion by simply inserting beyond the centers a perfect mirror [see Fig. 1(c)]. As this introduces a hard-wall boundary condition (BC) preventing $f$ from trespassing the right end, this way the transmission channel is in fact fully suppressed. Hence, $t_{\nu \mu} \equiv 0$ and (2) reduces to

$$
\rho^{\prime}=\mathbf{R} \rho \mathbf{R}^{\dagger},
$$

where now $\hat{R} \hat{R}^{\dagger}=\hat{R}^{\dagger} \hat{R}=\mathbb{1}$; that is, in the presence of the mirror $\hat{R}$ becomes a unitary gate. Thereby, for a monochro- (a)

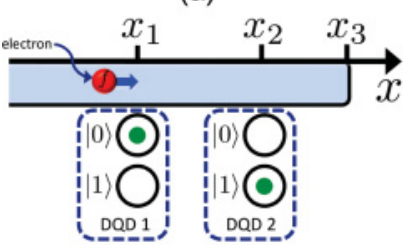

(b)

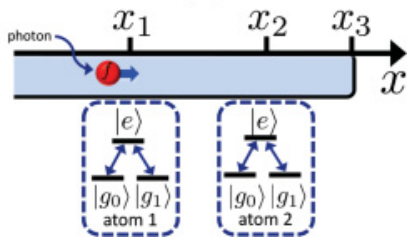

FIG. 2. (Color online) Setups implementing our scheme. (a) An electron along a quantum wire with double quantum dots (DQDs). (b) A photon along a waveguide with $\Lambda$-type atomlike systems. The right end of the wire or waveguide behaves as a perfect mirror. matic wave packet such gate is deterministically implemented whenever $f$ scatters from the centers. Remarkably, this holds regardless of the specific scattering potential. Rather, this affects only the type of achieved gate.

Having illustrated how a hard-wall BC guarantees the process unitarity, it is now natural to wonder whether there exist elastic one-channel scattering processes allowing for multiqubit gates that are universal for QC [1]. We show that this is indeed the case. With this aim, we first focus on a simple paradigmatic setting, setup $A$, comprising two spin- $\frac{1}{2}$ centers, that is, two qubits [1], each coupled to a massive particle embodying $f$. We identify a regime such that a CZ gate, universal for QC [1], naturally arises. We next address setup $B$, comprising multilevel atomlike systems and photons, which is within experimental reach in several scenarios [18-22]. We show that this can work as an effective emulator of setup A.

Setup A. This setting [see Fig. 2(a)] comprises two identical spin- $\frac{1}{2}$ scattering centers, 1 and 2 , lying along a semi-infinite wire along the $x$ axis at $x=x_{1}$ and $x=x_{2}$, respectively, each coupled to a scattering particle $f$ of mass $m$. The wire ends at $x=x_{3}$. Let $\left\{|0\rangle_{i},|1\rangle_{i}\right\}$ be an orthonormal basis for the $i$ th center $(i=1,2)$. In practice, one can consider a semiconducting quantum wire or a carbon nanotube [23] where an electron populating the lowest subband can undergo scattering from two double quantum dots (DQDs) [24] to which it is electrostatically coupled. As shown in Fig. 2(a), each single-electron DQD is in $|0\rangle(|1\rangle)$ if the upper (lower) dot is occupied, hence implementing an effective qubit [24] (tunneling between the upper and lower dots is negligible). Also, the electrostatic coupling is negligible for state $|1\rangle$. The Hamiltonian is thus modeled as (we set $\hbar=1$ throughout)

$$
\hat{H}=\frac{\hat{p}^{2}}{2 m}+\Gamma \delta\left(x-x_{1}\right)|0\rangle_{1}\left\langle 0\left|+\Gamma \delta\left(x-x_{2}\right)\right| 0\right\rangle_{2}\langle 0|,
$$

where $\hat{p}=-i d / d x$ is the momentum operator of $f$, while $\Gamma$ is the height of each contact potential scattering centered at $x=x_{i}(i=1,2)[25]$. Note that the scattering potential in (3) is dispersive because it cannot induce either 1 or 2 to flip between $|0\rangle$ and $|1\rangle$. Upon scattering, each initial SCs' state $\left|\alpha_{1} \alpha_{2}\right\rangle_{12}$ $\left(\alpha_{i}=0,1\right)$ simply but crucially picks up its own phase shift. It is trivially checked that for a given state $\boldsymbol{\alpha} \equiv\left\{\alpha_{1}, \alpha_{2}\right\} \hat{H}$ takes the effective form $\hat{H}_{\boldsymbol{\alpha}}=\frac{\hat{p}^{2}}{2 m}+\sum_{i=1,2} \Gamma \delta_{\alpha_{i} 0} \delta\left(x-x_{i}\right)$. The problem thus reduces to a particle scattering from spinless potentials. We label with $r_{\alpha}$ the $f$ 's reflection probability amplitude corresponding to $\hat{H}_{\alpha}$, where the subscript here specifies both the initial and final centers' state (these coincide owing to the dispersive interaction). Likewise, in the DQDs computational basis $\{|00\rangle,|01\rangle,|10\rangle,|11\rangle\} \mathbf{R}$ necessarily has the diagonal form $\mathbf{R}=\operatorname{diag}\left(\mathrm{r}_{00}, \mathrm{r}_{01}, \mathrm{r}_{10}, \mathrm{r}_{11}\right)$.

Adopting a standard procedure, to derive $r_{\alpha}$ we assume that $f$ is left-incoming and seek the stationary state $\Psi_{\alpha}(x)$ fulfilling $\hat{H}_{\alpha} \Psi_{\alpha}(x)=k^{2} /(2 m) \Psi_{\alpha}(x)$ of the form $\Psi_{\alpha}(x)=$ $\Psi_{\alpha+}(x)+\Psi_{\alpha-}(x)$ with (for simplicity we drop the dependance on $\boldsymbol{\alpha}$ whenever unnecessary)

$$
\begin{aligned}
\Psi_{+}(x)= & \frac{1}{\sqrt{2 \pi}}\left\{\theta(-x)+a_{1}\left[\theta(x)-\theta\left(x-x_{2}\right)\right]\right. \\
& \left.+a_{2} \theta\left(x-x_{2}\right)\right\} e^{i k x}
\end{aligned}
$$




$$
\begin{aligned}
\Psi_{-}(x)= & \frac{1}{\sqrt{2 \pi}}\left\{r \theta(-x)+b_{1}\left[\theta(x)-\theta\left(x-x_{2}\right)\right]\right. \\
& \left.+b_{2} \theta\left(x-x_{2}\right)\right\} e^{-i k x}
\end{aligned}
$$

where $k>0, \theta(x)$ is the Heaviside step function and we set $x_{1}=0$. Due to Eqs. (4) and (5), $\Psi_{+}(x)\left[\Psi_{-}(x)\right]$ represents the right-propagating (left-propagating) part of $\Psi(x)$. Note that $\Psi(x)$ is specified by the five $\boldsymbol{\alpha}$-dependent coefficients $\left\{r, a_{1}, b_{1}, a_{2}, b_{2}\right\}$. These are found by requiring that $\Psi(x)$ and its derivative with respect to $x \Psi^{\prime}(x)$ match the five BCs:

$$
\begin{gathered}
\Psi\left(x_{i}^{-}\right)=\Psi\left(x_{i}^{+}\right) \quad(i=1,2), \quad \Psi\left(x_{3}\right)=0, \\
\left.\Delta \Psi^{\prime}\right|_{x_{i}}=2 m \Gamma \delta_{\alpha_{i} 0} \Psi\left(x_{i}\right) \quad(i=1,2) .
\end{gathered}
$$

Equations (6) ensure the matching of the wave function at the centers' locations (first two) and the hard-wall BC owing to the end of the wire at $x=x_{3}$ (latter equation). Equations (7) are standard constraints on the discontinuity of $\Psi^{\prime}(x)$ at the centers' positions $\left.\Delta \Psi^{\prime}\right|_{x_{i}}=\Psi^{\prime}\left(x_{i}^{+}\right)-\Psi^{\prime}\left(x_{i}^{-}\right)$ due to the $\delta$ potentials in (3) [they are derived by integrating the Schrödinger equation (SE) corresponding to $\hat{H}_{\alpha}$ over an infinitesimal range across $x=x_{1}$ and $x=x_{2}$ ]. By solving the linear system (6)-(7) we end up with

$$
\begin{aligned}
r_{\alpha}= & -\exp \left\{2 i \operatorname { a r g } \left[\exp \left(i k x_{31}\right)+2 \gamma \delta_{\alpha_{2} 0}\left[\cos k x_{21}\right.\right.\right. \\
& \left.\left.\left.+\left(i-2 \gamma \delta_{\alpha_{1} 0}\right) \sin k x_{21}\right] \sin k x_{32}-2 \gamma \delta_{\alpha_{1} 0} \sin k x_{31}\right]\right\},
\end{aligned}
$$

where $x_{i j}=x_{i}-x_{j}$ and $\gamma=m \Gamma / k$. As expected, this yields $\left|r_{\alpha}\right|=1$ regardless of all the parameters and $\alpha$; namely, $f$ is reflected back with certainty. Consider the regime

$$
k x_{21}=n \pi, \quad k x_{32}=\left(n^{\prime}+1 / 2\right) \pi, \quad \gamma \gg 1,
$$

where $n$ and $n^{\prime}$ are arbitrary integers. Replacing Eqs. (9) in (8) and using $k x_{31}=k x_{32}+k x_{21}$, we end up with

$$
r_{00}=r_{01}=r_{10}=-r_{11}=-1,
$$

which yields the gate matrix $\mathbf{R}=\operatorname{diag}(1,1,1,-1)$ (up to an irrelevant global phase factor), that is, the well-known $\mathrm{CZ}$ gate [1]. This proves that a single scattering process can implement a universal TQG. Intuitively, unlike Ref. [17] here the geometry secures unitarity, leaving the physical parameters free to be tuned so as to match a CZ.

Setup B. In this setting [see Fig. 2(b)] $f$ is a photon propagating along a $1 \mathrm{D}$ waveguide, having geometry analogous to setup A, and scattering from two three-level atomlike systems. Each "atom" $i=1,2$ has a $\Lambda$-type energy-level configuration consisting of a twofold-degenerate ground doublet spanned by states $\left\{\left|g_{0}\right\rangle,\left|g_{1}\right\rangle\right\}$ and an excited state $|e\rangle$ [see Fig. 2(b)]. Hence, a two-photon Raman transition between $\left|g_{0}\right\rangle$ and $\left|g_{1}\right\rangle$ can occur through absorption and re-emission of a scattering photon. Assuming a linear photon dispersion law $\omega=v k$ with $\omega$ the photon energy and $v$ the group velocity, the free-field Hamiltonian in the waveguide reads [27,28] $\hat{H}_{f}=-i \sum_{d= \pm} \int d x v_{d} \hat{c}_{d}^{\dagger}(x) \partial_{x} \hat{c}_{d}(x)$ with $v_{+}=-v_{-}=v$ and $\hat{c}_{+}^{\dagger}(x)\left[\hat{c}_{-}^{\dagger}(x)\right]$ as the bosonic operator creating a right (left) propagating photon at $x$. The free atomic Hamiltonian reads $\hat{H}_{a}=\omega_{0} \sum_{i=1,2}|e\rangle_{i}\langle e|$, where $\omega_{0}$ is the energy gap between $|e\rangle$ and the ground doublet. The field- $i$ th atom coupling is modeled as [28] $\hat{H}_{f i}=J \int d x \delta\left(x-x_{i}\right)\left[\hat{c}(x) \hat{S}_{i}^{\dagger}+\right.$ H.c.] (under the usual rotating-wave approximation), where $\hat{c}(x)=$ $\hat{c}_{+}(x)+\hat{c}_{-}(x)$ annihilates a photon at $x$ regardless of its propagation direction, $J$ is the rate associated with each transition $\left|g_{j}\right\rangle_{i} \leftrightarrow|e\rangle_{i}(\forall j=0,1)$, and $\hat{S}_{i}^{\dagger}=\sum_{j}|e\rangle_{i}\left\langle g_{j}\right|$. The full Hamiltonian thus reads $\hat{H}=\hat{H}_{f}+\hat{H}_{a}+\hat{H}_{f 1}+\hat{H}_{f 2}$. It is convenient to use as the basis of each ground doublet the symmetric and antisymmetric combinations of the two ground states $\left|\phi^{ \pm}\right\rangle_{i}=\left(\left|g_{0}\right\rangle_{i} \pm\left|g_{1}\right\rangle_{i}\right) / \sqrt{2}$. As $\left|\phi^{-}\right\rangle_{i}$ is a dark state [28], that is, $\hat{S}_{i}^{\dagger}\left|\phi^{-}\right\rangle_{i}=0$, the atomic raising operator takes the effective form $\hat{S}_{i}^{\dagger} \equiv \sqrt{2}|e\rangle_{i}\left\langle\phi^{+}\right|$. Thus the Raman process does not couple $\left|\phi^{+}\right\rangle$and $\left|\phi^{-}\right\rangle$. It should be clear now that by taking $|0\rangle=\left|\phi^{+}\right\rangle$and $|1\rangle=\left|\phi^{-}\right\rangle$for each atom, as long as these are initially in the ground doublet, setup B in fact possesses all the key features of A. Indeed, if the $i$ th atom is in $|1\rangle_{i}=\left|\phi^{-}\right\rangle_{i}$ the corresponding potential $\hat{H}_{f i}$ vanishes. If in $|0\rangle_{i}$, it may undergo a second-order transition $|0\rangle_{i} \rightarrow|e\rangle_{i} \rightarrow|0\rangle_{i}$ so as to eventually pick up a phase shift once $f$ is scattered off. To make rigorous such considerations, we next prove that the reflection coefficients are again, with due replacements, given by Eq. (8) as for setup A.

$\hat{H}$ conserves the total number of excitations. Thus, following a standard approach [27,28], we seek one-excitation stationary states of the form

$$
\begin{aligned}
\left|\Psi_{\alpha}\right\rangle= & \sum_{d= \pm} \int d x \psi_{\alpha d}(x) \hat{c}_{d}^{\dagger}(x)|\mathrm{vac}\rangle\left|\alpha_{1} \alpha_{2}\right\rangle_{12} \\
& +\varepsilon_{1}|\mathrm{vac}\rangle\left|e \alpha_{2}\right\rangle_{12}+\varepsilon_{2}|\mathrm{vac}\rangle\left|\alpha_{1} e\right\rangle_{12}
\end{aligned}
$$

where $\psi_{\alpha \pm}(x)$ have a form analogous to Eqs. (4) and (5), thus being specified by parameters $\left\{r, a_{1}, a_{2}, b_{1}, b_{2}\right\},\left\{\varepsilon_{i}\right\}$ are excitedstate amplitudes, and $|\mathrm{vac}\rangle$ is the field vacuum state. Thus, for given $\alpha\left|\Psi_{\alpha}\right\rangle$ is specified by the seven complex amplitudes $\left\{r, a_{1}, a_{2}, b_{1}, b_{2}, \varepsilon_{1}, \varepsilon_{2}\right\}$ and obeys the SE $\hat{H}\left|\Psi_{\alpha}\right\rangle=v k\left|\Psi_{\alpha}\right\rangle$. Projecting this onto $c_{ \pm}^{\dagger}(x)|\mathrm{vac}\rangle\left|\alpha_{1} \alpha_{2}\right\rangle$ gives (we henceforth omit subscript $\boldsymbol{\alpha}$ )

$$
\mp i v \psi_{ \pm}^{\prime}(x)+\tilde{J} \sum_{i=1,2} \delta_{\alpha_{i} 0} \varepsilon_{i} \delta\left(x-x_{i}\right)=v k \psi_{ \pm}(x),
$$

where $\tilde{J}=\sqrt{2} J$ is the rate associated with the transition $|0\rangle \leftrightarrow|e\rangle$. Further projection of the SE onto $|\mathrm{vac}\rangle\left|e \alpha_{2}\right\rangle$ and $|\mathrm{vac}\rangle\left|\alpha_{1} e\right\rangle$ immediately yields that for each $i=1,2 \varepsilon_{i}=$ $\tilde{J} \delta_{\alpha_{i} 0} \psi\left(x_{i}\right) /\left(v k-\omega_{0}\right)$. Replacing these in Eq. (12), we are left with $\left\{\psi(x), \psi_{ \pm}(x)\right\}$ only

$$
\begin{aligned}
& \mp i v \psi_{ \pm}^{\prime}(x)+\tilde{J}^{2} /\left(v k-\omega_{0}\right) \sum_{\ell=1,2} \delta_{\alpha_{\ell} 0} \psi\left(x_{\ell}\right) \delta\left(x-x_{\ell}\right) \\
& =v k \psi_{ \pm}(x) .
\end{aligned}
$$

Subtracting now the equation for $\psi_{-}$from the $\psi_{+}$'s one yields $-i \psi^{\prime}(x)=k\left[\psi_{+}(x)-\psi_{-}(x)\right]$, which trivially entails that $-\left.i \Delta \psi^{\prime}\right|_{x_{\ell}}=k\left(\left.\Delta \psi_{+}\right|_{x_{\ell}}-\left.\Delta \psi_{-}\right|_{x_{\ell}}\right)$ holds as well for each $\ell=1,2$. Each $\left.\Delta \psi_{ \pm}\right|_{x_{\ell}}$ on the right-hand side of the above can be evaluated by integrating Eq. (13) over an infinitesimal interval across $x=x_{\ell}(\ell=1,2)$, which straightforwardly yields $\left.\Delta \psi_{ \pm}\right|_{x_{\ell}}=\mp(i / v) \tilde{J}^{2} /\left(v k-\omega_{0}\right) \delta_{\alpha_{\ell} 0} \psi\left(x_{\ell}\right)$. Thereby

$$
\left.\Delta \psi^{\prime}\right|_{x_{\ell}}=2 \frac{k}{v} \frac{\tilde{J}^{2}}{v k-\omega_{0}} \delta_{\alpha_{\ell} 0} \psi\left(x_{\ell}\right)(\ell=1,2) .
$$


This is identical to Eq. (7) once we set

$$
\Gamma=\frac{\tilde{J}^{2}}{v k-\omega_{0}}
$$

and note that, due to the parabolic dispersion law, in setup A $m=k / v$.

As $\psi(x)$, besides Eq. (14), must fulfill conditions analogous to Eqs. (6) due to the common geometry of setups A and B, we conclude that amplitudes $\left\{r_{\alpha}\right\}$ for setting $\mathrm{B}$ are identical to Eq. (8) with the effective mass and potential height given by $k / v$ and (15), respectively. In passing, note that $\left|r_{\alpha}\right|^{2}=1$, showing that if $f$ is absorbed it will be re-emitted with certainty (each atom behaves as an effective qubit encoded in $\left.\left\{\left|g_{0}\right\rangle,\left|g_{1}\right\rangle\right\}\right)$. It is now evident that setup B can be used as an emulator of A, thereby allowing for occurrence of the CZ gate [cf. Eq. (10)] under conditions (9). Interestingly, the requirement $\gamma=\Gamma / k \gg 1$ now in fact becomes the resonance condition $(\mathrm{RC}) v k \simeq \omega_{0}$. This agrees with [27], where it was shown that the reflectivity of an atomic scatterer becomes unitary in this limit. Also, although of a different nature, the first two requirements in Eq. (9) are RCs: The CZ gate thus stems from a combination of RCs. As anticipated, setup B can be experimentally implemented in several different ways, including photonic-crystal waveguides with defect cavities [18], semiconducting (diamond) nanowires with embedded QDs (nitrogen vacancies) [19,20], optical or hollow-core fibers interacting with atoms [21], and microwave (MW) transmission lines coupled to superconducting qubits [22]. In particular, highly efficient coupling between the dot and the fundamental waveguide mode has been recently achieved in tapered InP nanowires with embedded InAsP QDs [20]. Interestingly, it was recently shown [26] that a hard-wall BC analogous to ours [cf. Fig. 2(b)] can benefit MW photodetection. It is also worth mentioning that some features of our scheme are reminiscent of Refs. [29] and [30].

Gate working condition. In practice, the incoming $f$ has a narrow but finite uncertainty $\Delta k$ around a carrier wave vector $k_{0}$ fulfilling (9). As $\mathbf{R}$ is $k$ dependent, we assessed its resilience against deviations from $k_{0}$ by using the process fidelity $F$ as a figure of merit [31]. In a representative case, $\Delta k \lesssim 5 \% k_{0}$ yields $F \gtrsim 95 \%$. Also, $\Delta k$ affects the gate duration $\Delta \tau$ according to $\Delta \tau \sim 1 /(v \Delta k)$ [31], which entails a minimum time $\Delta \tau_{\min } \sim 10\left(v k_{0}\right)^{-1}$. If $T_{\mathrm{d}}$ is the system's decoherence time, the gate works reliably when $\Delta \tau_{\min } \ll T_{\mathrm{d}}$, and hence the working condition reads $T_{\mathrm{d}} \gg 10\left(v k_{0}\right)^{-1}$. This is matched in realistic instances [31].

Conclusions. We have shown a strategy to quasideterministically carry out multiqubit gates between static qubits through single flying buses scattering from them. This is effective without demanding postselection of any kind or iteration. The possibility of naturally implementing a universal $\mathrm{CZ}$ gate has been proven in two different setups including $1 \mathrm{D}$ photonic waveguides coupled to atomlike qubits. We believe this work can set a significant milestone for future advancements in the area of distributed QIP as well as in the emerging field of quantum optics in 1D waveguides. The design of a full quantum computing architecture, implementing single-qubit operations besides the proposed multiqubit gates, will be the subject of a future comprehensive work [32].

Acknowledgments. We acknowledge support from FIRB IDEAS (project RBID08B3FM), the National Research Foundation and Ministry of Education of Singapore, Leverhulme Trust, the EPSRC, the Royal Society, and the Wolfson Foundation.
[1] M. A. Nielsen and I. L. Chuang, Quantum Computation and Quantum Information (Cambridge University Press, Cambridge, UK, 2000).

[2] J. I. Cirac, P. Zoller, H. J. Kimble, and H. Mabuchi, Phys. Rev. Lett. 78, 3221 (1997); D. E. Browne, M. B. Plenio, and S. F. Huelga, ibid. 91, 067901 (2003); S. Bose, P. L. Knight, M. B. Plenio, and V. Vedral, ibid. 83, 5158 (1999); J. I. Cirac, A. K. Ekert, S. F. Huelga, and C. Macchiavello, Phys. Rev. A 59, 4249 (1999).

[3] Y. L. Lim, A. Beige, and L. C. Kwek, Phys. Rev. Lett. 95, 030505 (2005); S. D. Barrett and P. Kok, Phys. Rev. A 71, 060310 (2005); Y. L. Lim et al., ibid. 73, 012304 (2006).

[4] A. T. Costa Jr., S. Bose, and Y. Omar, Phys. Rev. Lett. 96, 230501 (2006).

[5] G. L. Giorgi and F. De Pasquale, Phys. Rev. B 74, 153308 (2006).

[6] K. Yuasa and H. Nakazato, J. Phys. A: Math. Theor. 40, 297 (2007); Y. Hida, H. Nakazato, K. Yuasa, and Y. Omar, Phys. Rev. A 80, 012310 (2009); K. Yuasa, J. Phys. A 43, 095304 (2010).

[7] M. Habgood, J. H. Jefferson, and G. A. D. Briggs, Phys. Rev. B 77, 195308 (2008); J. Phys.: Condens. Matter 21, 075503 (2009).
[8] F. Ciccarello et al., New J. Phys. 8, 214 (2006); J. Phys. A: Math. Theor. 40, 7993 (2007); F. Ciccarello, G. M. Palma, and M. Zarcone, Phys. Rev. B 75, 205415 (2007); F. Ciccarello, M. Paternostro, G. M. Palma, and M. Zarcone, ibid. 80, 165313 (2009); F. Ciccarello, M. Paternostro, M. S. Kim, and G. M. Palma, Phys. Rev. Lett. 100, 150501 (2008); F. Ciccarello, M. Paternostro, G. M. Palma, and M. Zarcone, New J. Phys. 11, 113053 (2009); F. Ciccarello et al., Solid State Sciences 11, 931 (2009); F. Ciccarello et al., Phys. Rev. A 82, 030302(R) (2010).

[9] A. De Pasquale, K. Yuasa, and H. Nakazato, Phys. Rev. A 80, 052111 (2009).

[10] Y. Matsuzaki and J. H. Jefferson, e-print arXiv:1102.3121.

[11] H. Schomerus and J. P. Robinson, New J. Phys. 9, 67 (2007).

[12] F. Buscemi, P. Bordone, and A. Bertoni, New J. Phys. 13, 013023 (2011)

[13] F. Ciccarello et al., Las. Phys. 17, 889 (2007).

[14] F. Ciccarello, M. Paternostro, M. S. Kim, and G. M. Palma, Int. J. Quant. Inf. 6, 759 (2008).

[15] K. Yuasa, D. Burgarth, V. Giovannetti, and H. Nakazato, New J. Phys. 11, 123027 (2009).

[16] D. D. Awschalom, D. Loss, and N. Samarth, Semiconductor Spintronics and Quantum Computation (Springer, Berlin, 2002). 
[17] G. Cordourier-Maruri et al., Phys. Rev. A 82, 052313 (2010).

[18] A. Faraon et al., Appl. Phys. Lett. 90, 073102 (2007).

[19] M. H. M. van Weert et al., Nano Lett. 9, 1989 (2009); J. Claudon et al., Nat. Photonics 4, 174 (2010); T. M. Babinec et al., Nat. Nanotechnol. 5, 195 (2010).

[20] M. E. Reimer et al., Nat. Commun. 3, 737 (2012); G. Bulgarini et al., Appl. Phys. Lett. 100, 121106 (2012).

[21] B. Dayan et al., Science 319, 1062 (2008); E. Vetsch et al., Phys. Rev. Lett. 104, 203603 (2010); M. Bajcsy et al., ibid. 102, 203902 (2009).

[22] A. Wallraff et al., Nature (London) 431, 162 (2004); O. Astafiev et al. Science 327, 840 (2010).

[23] D. Gunlycke et al., J. Phys.: Condens. Matter 18, S851 (2006); S. J. Tans et al., Nature (London) 386, 474 (1997).

[24] T. Hayashi, T. Fujisawa, H. D. Cheong, Y. H. Jeong, and Y. Hirayama, Phys. Rev. Lett. 91, 226804 (2003); T. Hayashi, D. G. Hasko, and D. A. Williams, ibid. 95, 090502 (2005);
J. Gorman, D. G. Hasko, and D. A. Williams, ibid. 95, 090502 (2005)

[25] The wire-end location $\left(x=x_{3}\right)$ does not enter Eq. (3). Following standard methods, the wire end is accounted for solely through a BC on the wave function [Eq. (6), second identity].

[26] B. Peropadre et al., Phys. Rev. A 84, 063834 (2011).

[27] J. T. Shen and S. Fan, Opt. Lett. 30, 2001 (2005); Phys. Rev. Lett. 95, 213001 (2005).

[28] D. Witthaut, and A. S. Sørensen, New J. Phys. 12, 043052 (2010).

[29] L.-M. Duan and H. J. Kimble, Phys. Rev. Lett. 92, 127902 Y (2004).

[30] Y.-F. Xiao, Z.-F. Han, and G.-C. Guo, Phys. Rev. A 73, 052324 (2006).

[31] See Supplemental Material at http://link.aps.org/supplemental/ 10.1103/PhysRevA.85.050305 for details on the working condition.

[32] F. Ciccarello et al. (unpublished). 